\title{
Determination of contact temperature on cutting disc during rock destruction
}

\author{
Vladimir Kuznetsov ${ }^{1}$, Elena Preis $^{1}$, Kirill Ananyev ${ }^{1}$, and Aleksander Ermakov ${ }^{1}$ \\ ${ }^{1}$ T.F. Gorbachev Kuzbass State Technical University, Kemerovo, Russia
}

\begin{abstract}
During the destruction of rocks by disc cutters the question of their operation without water cooling is arising. To implement this an approach to determining the temperature of the disk cutter is considered in the article. The calculations of heating of the disc cutter with various geometric parameters (diameter, sharpening angles, blunting edge radius) and geological conditions under different cutting conditions (cutting depth) are presented. The disc cutter temperature and comparison with a critical one is given. The results allow to increase wear resistance and thereby determine the modes of operation in which forced cooling of the cutting disc is not required.
\end{abstract}

\section{Introduction}

The most common cutting tool used in roadheaders and shearers is a cutting pick. Cutting picks are well proven in the destruction of weak and medium-strength rocks. At the same time, it was proven that when operating mining performs in difficult geological conditions (the presence of solid inclusions and highly abrasive rocks) it is advisable to destroy the rocks using disc cutters [1-4]. Different types of cutting drums equipped with such tools are shown in the fig.1.

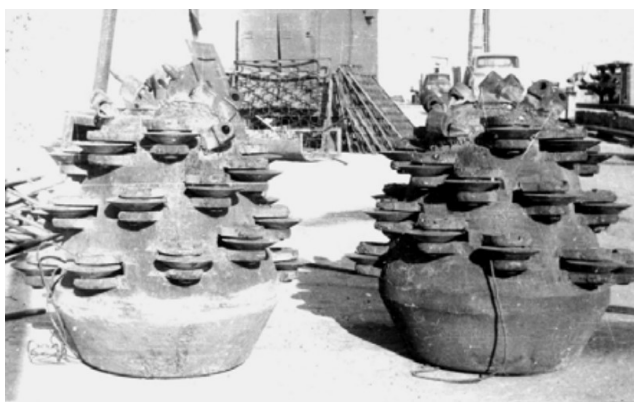

Fig. 1. Cutting drums equipped with disc cutters.

One of the advantages of cutting discs is that the destruction of the rocks occurs in large fractions without the formation of dust. That makes it possible to refuse to supply water under each disc and use only an external spraying system. However, the rejection of local spraying requires the thermal calculation of the disk. The purpose of this calculation is to check for exceeding the critical temperature at which the properties of the metal of the cutting disc can deteriorate. 


\section{Materials and Methods}

The determination of tool heating is truly relevant for metalworking and in this area has been studied in [5-8]. There are no detailed studies to determine the heating temperature of cutting disc during rock destruction have been identified. To determine the highest value of the contact temperature at the cutting disc, the formula for determining the temperature at the pick cutter with steady heat flow and free cooling is used as the basis:

$$
T_{u}=\frac{0,008 q_{3} \frac{\Delta_{i}}{\lambda_{r}} A_{3}^{S}}{1+0,223 A_{3} \frac{\lambda_{p}}{\lambda_{r}} \sqrt{\frac{\Delta_{i} V_{r}}{a_{p}}}},
$$

where $q_{3}$ - heat-flux density on a contact area, W/ $\mathrm{cm}^{2}, V_{r}-$ cutting speed, $\mathrm{m} / \mathrm{min}$, $S$ - surface contact area of cutting disc and rock, $\mathrm{mm}^{2}, \Delta_{i}$ - linear wear of the tool (for cutting disc is equivalent to the depth of cut $h), \mathrm{mm}, \lambda_{r}$ - heat conduction coefficient of cutting disc, $\mathrm{W} /(\mathrm{cm} \cdot \mathrm{K}), \lambda_{p}$ - heat conduction coefficient of rock, $\mathrm{W} /(\mathrm{cm} \cdot \mathrm{K}), \alpha_{p}-$ thermal diffusivity coefficient of rock, $\mathrm{cm}^{2} / \mathrm{c}$.

Heat-flux density on a contact area is

$$
q_{3}=\frac{100 P_{f} V_{r}}{60 S}=\frac{1,67 P_{f} V_{r}}{S},
$$

where $P_{f}$ is the friction force on a contact area, N.

$$
V_{r}=2 V \sqrt{\frac{h}{2 R}},
$$

where $V$ is the feeding speed of cutting disc, $\mathrm{m} / \mathrm{min}, \mathrm{R}$ is the cutting disc radius, $\mathrm{mm}$.

The friction force is determined by Equation

$$
P_{f}=0,25 \mu P_{k} S
$$

where $\mu$ is the cutting resistance coefficient, $P_{k}$ - contact strength of rock, MPa.

$$
\mu=\mu_{f}+0,24 h
$$

where $\mu_{f}=\frac{P \cdot D}{d \cdot G} \approx 0,4$ is the coefficient of the disk friction on the rock, $P$ - breakaway force, $\mathrm{kgf}, D$ - cutting disc diameter, $\mathrm{mm}, d$ - cutting disc joint-pin diameter, mm, $G$ - loading force, $\mathrm{kgf}$.

The function $A_{3}^{S}$ can be determined by equation

$$
A_{3}^{S} \approx 4,81 F_{S}(1, \varphi)+\frac{1440}{\varphi} \lg \frac{b}{\Delta_{i}},
$$

where $b$ is the average width of the area contact area of cutting disc and rock, mm.

$$
b=\rho+(h-\rho) \operatorname{tg}\left(\varphi_{1}+\varphi_{2}\right),
$$

where $\rho$ is blunting cutter disc edge radius, $m m, \varphi_{1}$ and $\varphi_{2}$ angles of inclination of generatrix to the axial section for cutting disc with asymmetric cutting-point angles (fig. 2). 


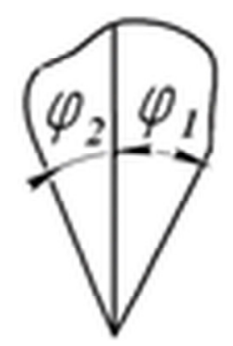

Fig. 2. Biconical cutting disc with asymmetric angles.

The use of cutting discs with asymmetric cutting-point angles is due to theoretical and experimental studies carried out at the Department of Mining Machines and Complexes of KuzSTU with the destruction of rocks with a strength of more than 8 units on a scale of M.M. Protodyakonov.

To determine the temperature on the tool according to equation (1), it is necessary to determine the contact area of the cutting disc with the rock $S$. We introduce the coordinate system and find the coordinates of points A and B (fig. 3). The origin will relate to the center of the disc of radius $\mathrm{R}$.

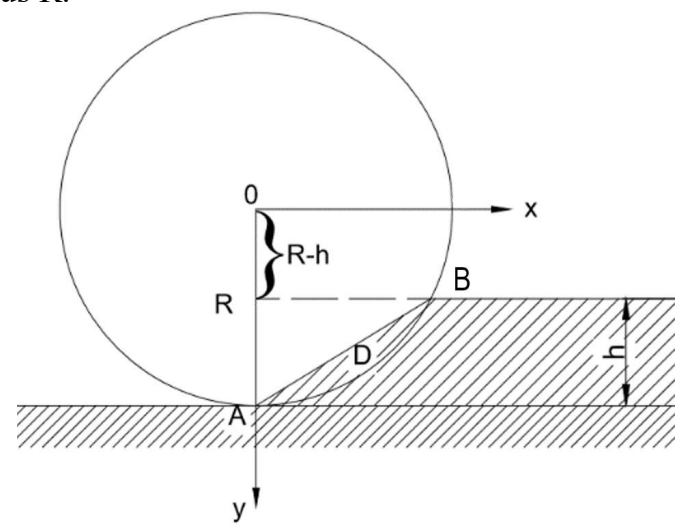

Fig. 3. Determination of the boundaries of the contact area.

Point A lies on a circle and has coordinates $A(O, R)$. For point B coordinate $y=R-h$, therefore $x=\sqrt{R^{2}-y^{2}}=\sqrt{R^{2}-(R-h)^{2}}=\sqrt{2 R h-h^{2}}$ and then the coordinates of the point $B\left(\sqrt{2 R h-h^{2}}, R-h\right)$.

The equation of the line $A B$ will look like this:

therefore, $y$ will be equal

$$
\frac{x-0}{\sqrt{2 R h-h^{2}}}=\frac{y-R}{R-h-R}
$$

$$
y=\frac{-h x}{\sqrt{2 R h-h^{2}}}+R \text {. }
$$

The contact area of the disk cutter with the rock is part of the conical surface, cut off by a plane

$$
y+\frac{h x}{\sqrt{2 R h-h^{2}}}-R=0 .
$$

Given that the disc cutter is two conical surfaces inclined at different angles to the axial section, it is necessary to determine the contact area for the angle $\varphi$ (fig. 4). 


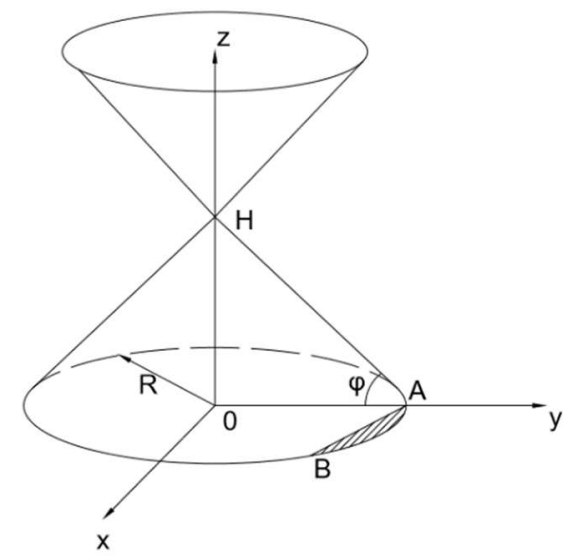

Fig. 4. Determination of contact area.

The conical surface is described by the equation $\frac{\left(z-z_{0}\right)^{2}}{c^{2}}=\frac{\left(x-x_{0}\right)^{2}}{a^{2}}+\frac{\left(y-y_{0}\right)^{2}}{b^{2}}$ and must pass through the points $(O, R, O) ;(R, O, O) ;(O, O, H)$, where $H=R \tan \varphi$. In this case, there will be $(z-H)^{2}=\frac{H^{2}}{R^{2}}\left(x^{2}+y^{2}\right)$. Therefore

$$
z=-\sqrt{\frac{H^{2}}{R^{2}}\left(x^{2}+y^{2}\right)}+H=-\frac{H}{R} \sqrt{x^{2}+y^{2}}+H .
$$

The surface area above segment $D$ (fig. 2) is determined by the surface integral

$$
S=\iint_{D} \sqrt{1+z_{x}^{\prime 2}+z_{y}^{\prime 2}} \mathrm{~d} x \mathrm{~d} y
$$

where $z_{x}^{\prime}=\frac{-H_{x}}{R \sqrt{x^{2}+y^{2}}} ; z_{y}^{\prime}=\frac{-H_{y}}{R \sqrt{x^{2}+y^{2}}}$.

Then the radical expression in equation (12) is transformed as follows:

$$
1+z_{x}^{\prime 2}+z_{y}^{\prime 2}=1+\left(\frac{H}{R}\right)^{2}=1+(\tan \varphi)^{2}
$$

where $\varphi=\varphi_{1}+\varphi_{2}$ is angle to the axial section, and expression (12) will look like this:

$$
\begin{aligned}
S=\sqrt{1+(\tan \varphi)^{2}} \iint_{D} \mathrm{~d} x \mathrm{~d} y=\sqrt{1+(\tan \varphi)^{2}} \int_{0}^{\sqrt{2 R h-h^{2}}} \mathrm{~d} x \int_{\frac{-h x}{\sqrt{2 R h-h^{2}}}}^{\sqrt{R^{2}-x^{2}}} \mathrm{~d} y= \\
=\sqrt{1+(\tan \varphi)^{2}} \int_{0}^{\sqrt{2 R h-h^{2}}} \mathrm{~d} x\left(\sqrt{\left(R^{2}-x^{2}\right.}+\frac{h x}{\sqrt{2 R h-h^{2}}}-R\right)= \\
=\sqrt{1+(\tan \varphi)^{2}}\left(\frac{x}{2} \sqrt{\left(R^{2}-x^{2}\right.}+\frac{R^{2}}{2} \arcsin \left(\frac{x}{R}\right)+\frac{h x^{2}}{2 \sqrt{2 R h-h^{2}}}-\right. \\
-R x)\left.\right|_{0} ^{\sqrt{2 R h-h^{2}}}= \\
=\sqrt{1+(\tan \varphi)^{2}}\left(\frac{R^{2}}{2} \arcsin \frac{\sqrt{2 R h-h^{2}}}{R}-\frac{R}{2} \sqrt{2 R h-h^{2}}\right) .
\end{aligned}
$$

Considering that the asymmetric disc cutter has angles of inclination to the axial section $\varphi_{1}$ and $\varphi_{2}$, the total, the bilateral surface area will be equal to

$$
S=\frac{R}{2}\left(\sqrt{1+\operatorname{tg}^{2} \varphi_{1}}+\sqrt{1+\operatorname{tg}^{2} \varphi_{2}}\right)\left(R \arcsin \frac{\sqrt{2 R h-h^{2}}}{R}-\sqrt{2 R h-h^{2}}\right)
$$

Thus, the contact area depends on the main parameters of the disc cutter: $\varphi_{1}, \varphi_{2}, R$, and the depth of cut $h$. 


\section{Results}

To analyze the obtained equations, we determine the temperature of the disc cutter heating for certain conditions and parameters indicated in the Table 1.

Table 1. Parameters for calculation.

\begin{tabular}{|l|l|l|}
\hline \multicolumn{1}{|c|}{ Parameter } & \multicolumn{1}{c|}{ Value } & \multicolumn{1}{c|}{ Remark } \\
\hline$R, \mathrm{~mm}$ & 150 & - \\
\hline$F_{c}$ & 3,259 & $\begin{array}{l}\text { for angle } \varphi=40^{\circ} \text { (according to A.N. } \\
\text { Reznikov) }\end{array}$ \\
\hline$\alpha_{p}, \mathrm{~cm}^{2} / \mathrm{s}$ & 122 & sandstone (average value) \\
\hline$\lambda_{p}, \mathrm{~W} /(\mathrm{cm} \cdot \mathrm{K})$ & 2,56 & sandstone (average value) \\
\hline$\lambda_{r}, \mathrm{~W} /(\mathrm{cm} \cdot \mathrm{K})$ & 55,6 & low-carbon steel \\
\hline$\varphi_{1}, \mathrm{deg}$ & 18 & - \\
\hline$\varphi_{2}, \mathrm{deg}$ & 22 & - \\
\hline$\rho, \mathrm{mm}$ & 2 & must be at least 1.5 \\
\hline
\end{tabular}

The graphs below (fig. 5) show the results of temperature calculation depending on the depth of cut $T_{u}=\mathrm{f}(h)$ for different contact strength of rock $P_{k}$ and feed rate $V$.
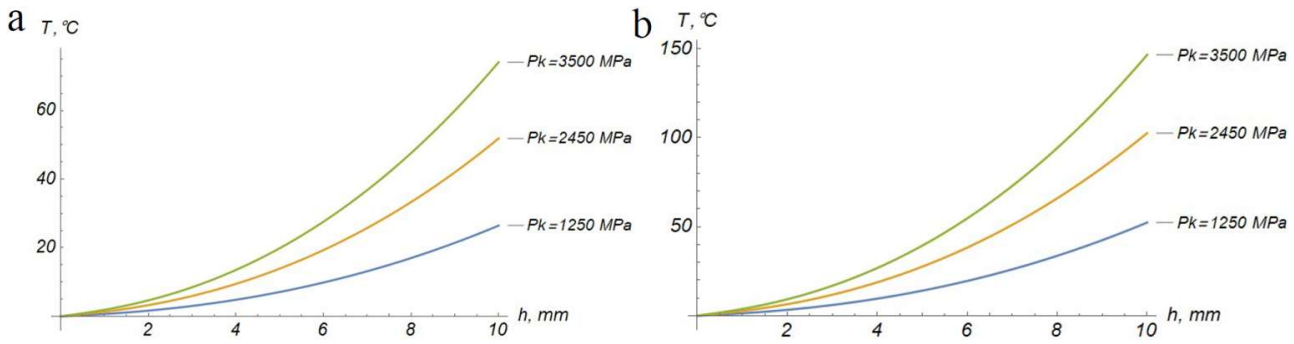

Fig 5. Graph of temperature versus depth of cut for feed rates $V=5 \mathrm{~m} / \mathrm{min}$ (a) and $10 \mathrm{~m} / \mathrm{min}(\mathrm{b})$.

\section{Conclusion}

The presented calculations of heating the disc cutter with various geometric parameters (diameter, sharpening angles, blunting edge radius) and geological conditions under different cutting conditions (cutting depth) allow us to determine the disc cutter temperature and compare it with a critical one that affects the structure and properties of the disc cutter material, which could lead to a decrease in wear resistance, and thereby determine the modes of operation in which forced cooling of the cutting disc is not required.

\section{References}

1. Z. Liu, W. Nie, H. Peng, S. Yang, D. Chen, Q. Liu, Min. Mach., 350, 62-80 (2019)

2. A. Khoreshok, L. Mametyev, V. Nesterov, A. Tsekhin, A. Borisov, MATEC Web Conf., 297 (2019)

3. K. Kotwica, P. Małkowski, Energies, 18 (2019)

4. A. Khoreshok, L. Mametyev, V. Nesterov, A. Tsekhin, A. Borisov, MATEC Web Conf., 297 (2019)

5. W. Baohai, C. Di, H. Xiaodong, Z. Dinghua, T. Kai, Chinese Journal of Aeronautics, 29(6), 1788-1794 (2016) 
6. G. Buyalich, K. Buyalich, M. Byakov, E3S Web Conf., 21, 03018 (2017)

7. B. P. Nedić, M. D. Erić, Cutting temperature measurement and material machinability, Thermal Science, 18, 259-268 (2014)

8. T. Ueda. Cutting Temperature (Springer, Berlin, 2014) 